\title{
Data Acquisition Systems for Magnetic Shield Characterization
}

\author{
Leopoldo Angrisani ${ }^{1}$, Mirko Marracci², \\ Bernardo Tellini ${ }^{2}$ and Nicola Pasquino ${ }^{3}$ \\ 'University of Naples "Federico II", Department of Computer Science and Control Systems \\ ${ }^{2}$ University of Pisa, Department of Electrical Systems and Automation \\ "University of Naples "Federico II", Department of Electrical Engineering
}

Italy

\section{Introduction}

The purpose of an EM shield is to prevent undesired electromagnetic coupling between an EM source and a susceptible EM device. The mechanism of electromagnetic coupling is by radiation, inductive and capacitive coupling. The radiation of energy by electromagnetic waves characterizes the EM coupling in the far field region, while inductive (or lowimpedance) and capacitive (or high-impedance) couplings are the driving mechanism of coupling in the near field region. In all the cases, a complete analysis of the shielding performances of an EM shield should require a full-wave solution of the electromagnetic problem. This means to solve a classical eddy current problem and in presence of magnetically permeable materials the analysis shall combine the Maxwell's equations with the magnetic properties of the shield.

Numerous studies of shielding problems can be found in the literature showing how this topic has been the subject of great interest to the scientific community for many years (Moser; 1988; Schelkunoff; 1943; Schulz et al.; 1988). Several analytical and numerical techniques have been developed during the years especially with the advent and proliferation of the electronic devices.

Different materials with a wide range of electrical and magnetic properties are used for shielding applications. As a matter of fact, an accurate knowledge of the electromagnetic properties of the material is an important task for a correct modeling and design phase. Further, shields have different shapes and contain apertures and all these parameters influence the shielding effectiveness, thus the electromagnetic susceptibility of the combined shieldelectronic equipment system.

In a first part, this chapter reports on basic aspects of the shielding theory and standard measurement methods of shielding effectiveness in the frequency and time domain. Concepts and measurement techniques are discussed with reference to basic shield configurations and following the relevant standards (IEEE Std 299; 2006). A basic configuration of the experimental setup and instrumentation chain for shielding effectiveness measurements is reported. 
In the second part, we focus on the magnetic shields that received most of the attention for shielding low-frequency magnetic fields (Celozzi \& D'Amore; 1996; Hoburg; 1995; Sergeant et al.; 2006).

The case of a circular loop magnetically coupled to an indefinite conducting plate and that of a hollow ring shield placed coaxially around a circular loop are treated to support our discussion (Di Fraia et al.; 2009).

Then, we conclude with a discussion on the characterization of magnetic materials. As better discussed in the chapter, an accurate knowledge of the magnetic characteristics of a magnetic material is at the basis for an accurate study and measurement of the shielding effectiveness of a magnetic shield. Experimental setups and instrumentation chain commonly adopted for magnetic materials characterization are shown.

\section{Shielding theory}

The shielding effectiveness (SE) is defined as the ratio of the signal received (from a transmitter) without $(w o)$ the shield, to the signal received inside the shield $(w)$. Alternatively, SE is defined as the insertion loss when the shield is placed between the transmitting antenna and the receiving antenna (IEEE Std 299; 2006). Thus, SE can be defined as:

$$
S E=20 \log \frac{\left|H_{i}\right|}{\left|H_{t}\right|}
$$

where $H_{i}$ and $H_{t}$ are the incident and transmitted magnetic field.

Time-varying electromagnetic fields in conducting media are driven by diffusion (or diffusion-like) equations. The analysis of electromagnetic field diffusion in conducting media is, in practice, the analysis of eddy currents in those conductors. A basic knowledge and modeling of the skin effect and the eddy currents are necessary so that efficient measures are performed to characterize the EMC parameters of a shield.

Shielding problems are generally approached by the scientific community through the solution of the vector wave equation or through the application of the Schelkunoff's theory (Moser; 1988). The use of impedance concept for dealing with the theory of the electromagnetic shielding was first introduced by Schelkunoff and therefore it is known as the Schelkunoff's theory (Schelkunoff; 1943).

An extended analysis of various basic eddy current problems can be found in (Tegopoulos \& Kriezis; 1985). Other treatments are reported in (Moser; 1988; Schulz et al.; 1988). In particular, an interesting study case is that of a loop antenna coupled to an indefinite conducting plate. A schematic representation of the problem is shown in Fig. 1.

The problem can be solved analytically and adopted as a benchmark for other analytical, numerical or experimental methods. As written in (IEEE Std 299; 2006) the shielding effectiveness can be related to the ratio of the voltage induced along the receiving loop without the shield, to that received in presence of the shield. Thus eq. (1) can be written as:

$$
S E=20 \log \frac{\left|V_{w o}\right|}{\left|V_{w}\right|}
$$

It is worth to say that in presence of nonuniform field distribution over the shield the SE parameter can vary significantly, depending on the adopted loop size. As a practical 


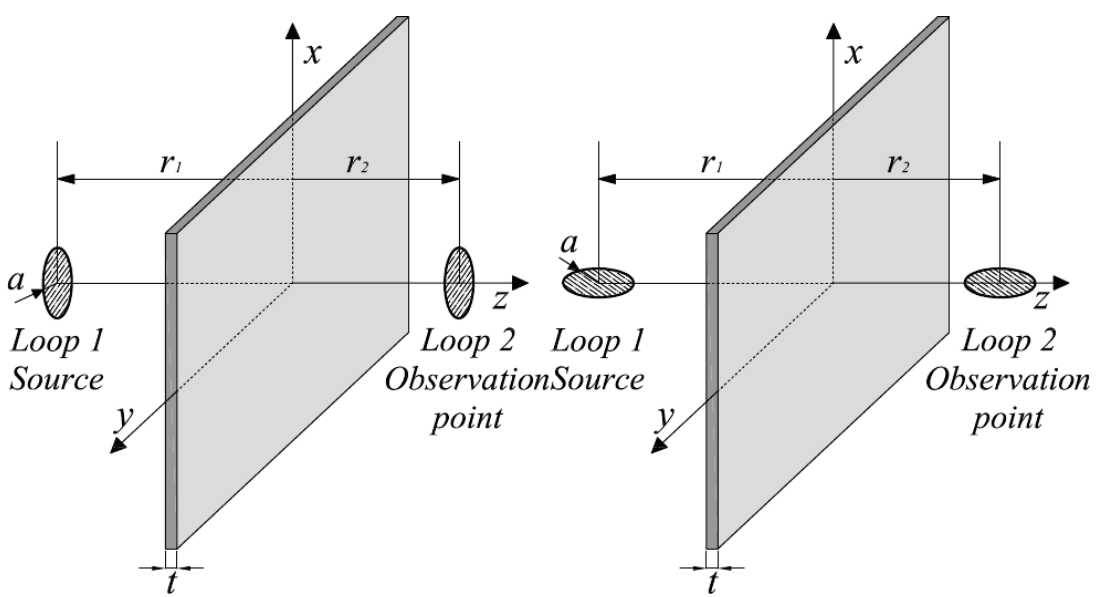

Fig. 1. Magnetic field loop source close to indefinite conducting plate.

consideration, it can be often the case that the geometry of the shield and the dimensions of the electronic device under test do not make it possible to place the chosen loop probe in the desired position and, as a consequence, to correctly perform the SE measurement. In some cases, this problem is solved by the use of smaller-size magnetic sensors. However, the averaging problem still stands and the estimation of the SE could be more properly defined by averaging the field over a spatial region better representative of the "victim".

As written in the previous text, an effective method for shielding problem consists in the application of the Schelkunoff's theory. Here, we limit to recall some basic aspects of this approach with reference to the case of a plane wave incident on an indefinite conducting lamination, and to the previous case of a loop coupled to an indefinite plate.

Due to the impedance discontinuity at the air-metal and metal-air boundaries and to the diffusion equation governing the field inside the shield, part of the energy is reflected at the two interfaces and part absorbed by the shield turning into heat energy. It is common notation to express the overall SE as the sum of three separate contributions:

$$
S E=R+A+M
$$

where all the terms are expressed in $\mathrm{dB}$ and $R$ represents the reflection losses at the left and right interfaces, $A$ is the term of the absorption losses, while $M$ is an additional terms due to multiple reflection effects. In the case of Fig. 2, SE can be analytically evaluated and its expression is:

$$
S E=20 \log _{10}\left|\frac{\left(\eta_{0}+\eta\right)^{2}}{4 \eta_{0} \eta}\right|+20 \log _{10}\left|e^{t / \delta}\right|+20 \log _{10}\left|1-\left(\frac{\eta_{0}-\eta^{2}}{\eta_{0}+\eta}\right) e^{t / \delta} e^{-i 2 \beta t}\right|
$$

where it is possible to recognize the reflection $R$, absorption $A$ and mutual reflection $M$ terms (Paul; 1992). $\eta_{0}$ and $\eta$ represent the intrinsic air and shield impedance, respectively. The Schelkunoff's theory can be extended to the case of shield in the near field region substituting the wave impedance of the magnetic field source in place of the impedance $\eta_{0}$ of the previous expressions. As well known from the theory, the wave impedance of a magnetic dipole can be expresses as: 


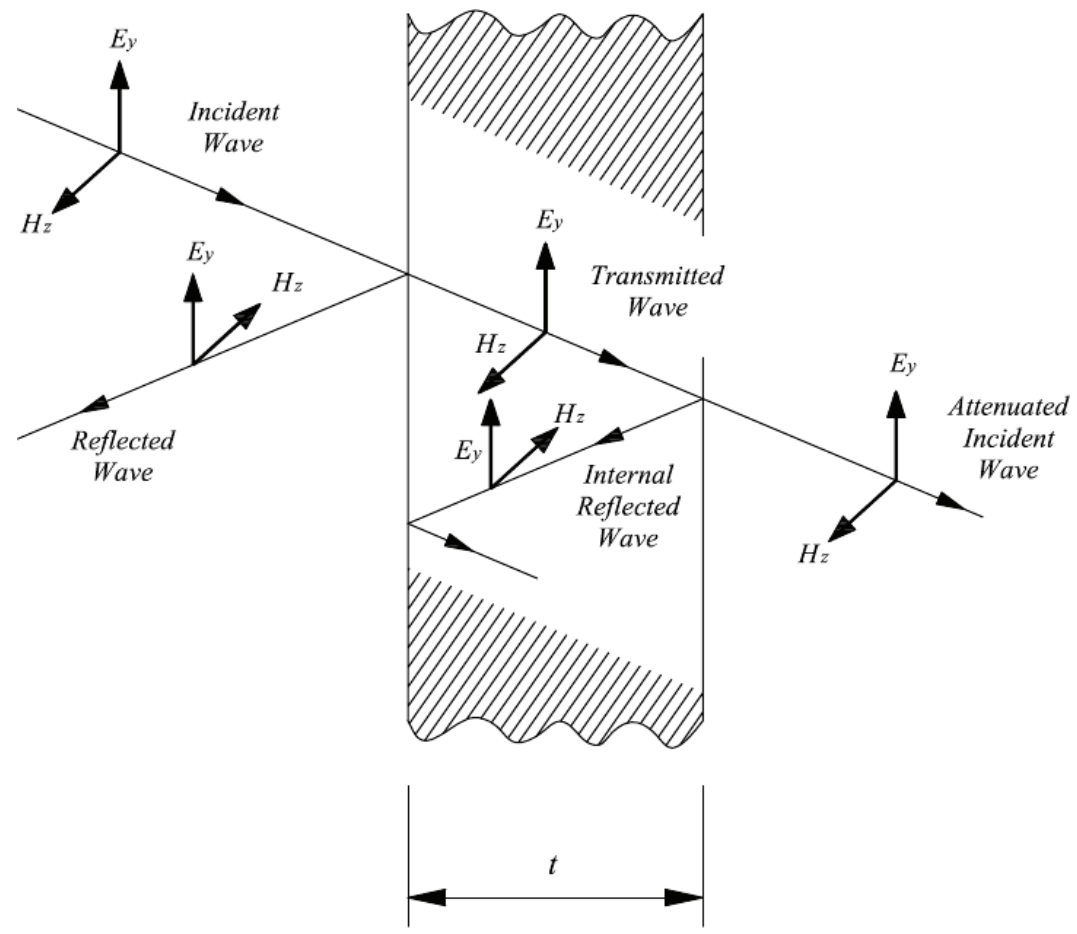

Fig. 2. Shielding effectiveness: plane wave incident on an indefinite conducting plate.

$$
\eta_{w}=\frac{E_{\phi}}{H_{\theta}}-\eta_{0} \frac{i /\left(\beta_{0} r\right)+1 /\left(\beta_{0} r^{2}\right)}{i /\left(\beta_{0} r\right)+1 /\left(\beta_{0} r^{2}\right)-i /\left(\beta_{0} r^{3}\right)}
$$

Thus, neglecting wave divergence in traversing our physically thin shield we can estimate the shielding effectiveness of the plate by replacing $\eta_{w}$ for $\eta_{0}$ in eq. (4).

\section{Shielding effectiveness measurement}

According to the IEEE 299 standard the range measurement frequencies for shielding effectiveness evaluation are those reported in Table 1.

The measurements shall be made in accordance to specific relevant positions among the transmitting, receiving antennas and shield. In particular, performing measurement in the low frequency range, loops shall be spaced each one by $0.3 \mathrm{~m}$ from the respective shielding barrier and coplanar in a plane perpendicular to the wall, ceiling, or other surface being measured (IEEE Std 299; 2006). A typical configuration is shown in Fig. 3.

As a basic concern of the electromagnetic compatibilty, measurements should be oriented to detect the worste case in order to prevent as much as possible disturbances to electronic equipments from electromagnetic interferences (EMI). According to such a fundamental rule, one loop (typically the transmitting antenna) is kept in a fixed position, while the receiving loop is reoriented and physically swept searching for the worst condition. The maximum reading of the receiver is adopted for evaluating the SE. 


\begin{tabular}{|c|c|}
\hline ExtendedFrequencyRange & Antenna Type \\
\hline $50 \mathrm{~Hz}-16 \mathrm{MHz}$ & Small loop \\
\hline $20 \mathrm{MHz}-100 \mathrm{MHz}$ & Biconical \\
\hline $100 \mathrm{MHz}-300 \mathrm{MHz}$ & Dipole \\
\hline $0.3 \mathrm{GHz}-1 \mathrm{GHz}$ & Dipole \\
\hline $1 \mathrm{GHz}-100 \mathrm{GHz}$ & Horn \\
\hline
\end{tabular}

Table 1. Range Measurement Frequencies for SE.

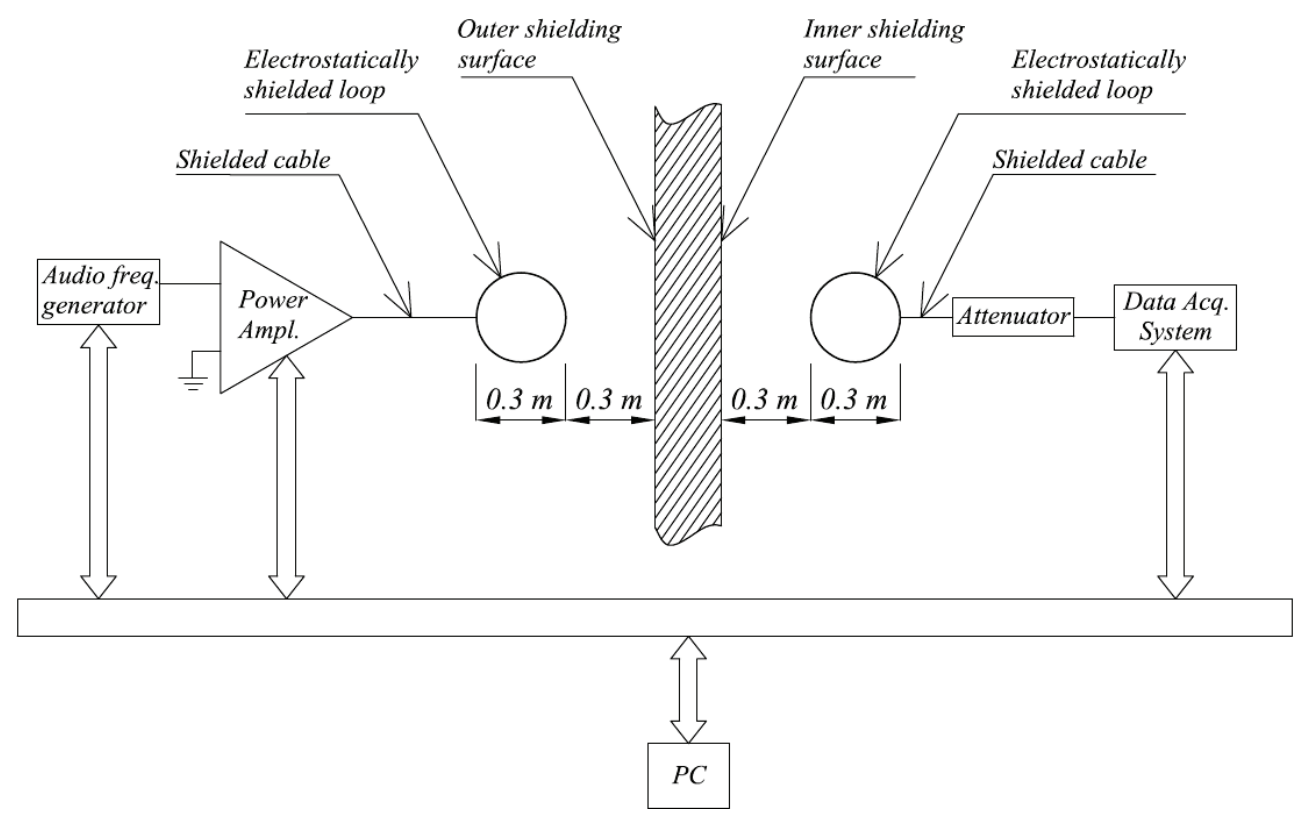

Fig. 3. Test configuration of SE measurements in the low frequency range.

Measurement data obtained following the previous procedure are converted in SE values through the following mathematical relations that vary vs. the operating frequency range:

$$
\begin{gathered}
S E_{H}=20 \log _{10}\left|\frac{H_{1}}{H_{2}}\right| \text { or } S E_{H}=20 \log _{10}\left|\frac{V_{1}}{V_{2}}\right|(50 \mathrm{~Hz}-20 \mathrm{MHz}) \\
S E_{E}=20 \log _{10}\left|\frac{E_{1}}{E_{2}}\right| \\
S E_{P}=20 \log _{10}\left|\frac{P_{1}}{P_{2}}\right|
\end{gathered}
$$

Many parameters such as the electromagnetic environment, the characteristics of the test site, the instrumentation chain itself, the positioning of the antennas participate at determining the measurement uncertainty of SE. The IEEE Std 299 reports that uncertainty 
in the measurement of SE is not required, even if it is recommended that a measurement uncertainty analysis be performed on each set of measurements and discussed in the final report. In addition, the IEEE Std 299 makes reference to the standards and technical notes relevant to the evaluation and expression of the uncertainty in measurement (NIST TN 1297; 1994).

\subsection{SE time and frequency domain measurements: data acquisition systems}

The procedure set in Standard IEEE Std 299 (2006) to test shielding effectiveness is basically a frequency domain technique, where a single tone within the test band is generated at a time and its amplitude is measured with and without the shield. A typical automated test and data acquisition procedure would therefore require repetitive execution of the following steps: firstly, the generator is set at a frequency and the signal is applied to the transmitting antenna; secondly, the receiver is tuned at the same frequency as the generator and the amplitude of the received signal is stored. Then the generator is set to the next frequency and the procedure goes on so to test all the frequency band. The time requirements for such a procedure to be run over the entire test band call for alternative methods and systems for shielding effectiveness measurement. In the following two proposals are presented.

\subsubsection{Time-Frequency Representation}

Time-Frequency Representations (TFR's) map a one-dimensional signal of time, $s(t)$, onto a two-dimensional function of time and frequency, $T_{s}(t, f)$ Hlawatsch et al (1992). A signal, as a function of time, may be considered as a representation with perfect time resolution. In contrast, the magnitude of the Fourier Transform (FT) of the signal may be considered as a representation with perfect spectral resolution but with no time information because the magnitude of the FT conveys frequency content but it fails to convey when, in time, different events occur in the signal. TFR's provide a bridge between these two representations in that they provide some temporal information and some spectral information simultaneously. In particular, most TFR's are "time-varying spectral representations," which are conceptually similar to a musical score with time running along one axis and frequency along the other. The values of the TFR surface above the timefrequency plane give an indication as to which spectral components are present at what times. Thus, TFR's are useful for the representation and analysis of signals containing one or more time-varying frequencies. One form of TFR (or TFD) can be formulated by the multiplicative comparison of a signal with itself, expanded in different directions about each point in time. Such representations and formulations are known as quadratic TFR's or TFD's because the representation is quadratic in the signal. One such representation is the WignerVille Distribution:

$$
T_{s}(t, f)=\int_{-\infty}^{+\infty} s(t-\tau / 2) s^{*}(t+\tau / 2) e^{-j 2 \pi f \tau} d \tau,
$$

The cross-terms caused by their bilinear structure may be useful in some applications such as classification as the cross-terms provide extra detail for the recognition algorithm. However, in some other applications, as for example the shielding effectiveness measurement, these cross terms may produce misinterpretations and they would need to be reduced. One way to do this is obtained by comparing the signal with a different function. Such resulting representations are known as linear TFR's because the representation is linear in the signal. The windowed Fourier transform, also known as the Short-Time Fourier 
Transform (STFT) localizes the signal by modulating it with a window function $h(\cdot)$, before performing the Fourier transform to obtain the frequency content of the signal in the region of the window. Its expression is:

$$
T_{s}(t, f ; h)=\int_{-\infty}^{+\infty} s(u) h^{*}(u-t) e^{-j 2 \pi f u} d u .
$$

In a digital implementation of a TFR, the obtained results are typically the squared values of the discrezited version of the aforementioned two-dimensional function, $T_{s}(n, v)$, the discrete variables, $n$ and $v$, represent, respectively, the time and frequency. These values are collected in a matrix. Generally, row index is connected to frequency, while column index represents time. By visualizing the matrix along a time-frequency plane, it can directly be observed how the power spectral contents of the analyzed signal evolve versus time. So, shielding effectiveness measurements can automatically be carried out by simply manipulating the coefficients of the matrix.

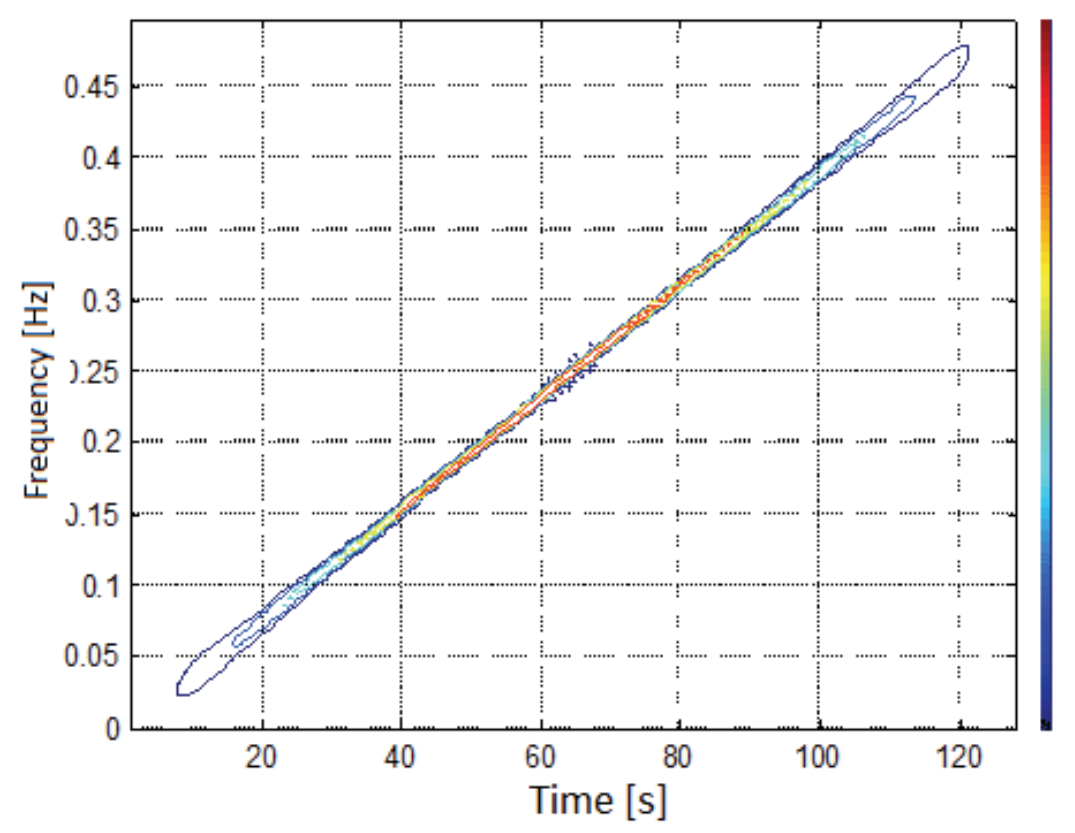

Fig. 4. Typical Time-Frequency Representation.

TFR's are often used for parameter's estimation Angrisani et al. (2002) and system testing Angrisani et al. (2000). Figure 4 shows a typical time-frequency representation for the response to an FM signal. This is what is expected to be seen when such techniques are applied to SE measurements: the whole frequency band in Table 1 is divided into sub-bands, then an FM spanning each sub-band is generated, and the response is first digitized by a data acquisition system with proper vertical resolution and sample rate, and then suitably processed in order to construct the desired time-frequency representation. The advantage 
over a frequency domain test is the capability of acquiring the response to more frequencies at the same time. Furthermore, unlike the classical approach such a methodology can be used even to characterize non-linear materials, as the analysis in the joint time-frequency domain can localize also harmonics and non-stationary components whereas a tone-by-tone investigation couldn't.

\subsubsection{Pulsed signal characterization}

Because of the wide frequency content of a pulse, it can be used to test the shielding properties of a material over an interval of frequencies. This is indeed quite a trivial task in terms of system requirements, given the excellent performance of both pulse generators and acquisition systems available on the market. The same envelope can be used to modulate different carriers so that the whole investigation band can be tested in different steps. With this approach, even a single pulse can be generated: due to the storing capability of modern digital oscilloscopes, by setting a pre-trigger acquisition mode with a one-shot trigger mode, the transient can be acquired and stored, and processed later (even off-line) by means of an FFT algorithm to detect the response of the shielding material to each frequency contained in the transmitted pulse. Again, unlike the direct frequency-domain analysis, this methodology can be used even in presence of dispersive or generally non-linear materials, given its nature of being a test for a packet of frequencies at the same time.

\section{Magnetic shields}

Magnetic field shielding at low and extremely low frequency (ELF) is a subject of particular interest for the industrial and scientific communities. Typical applications include medical instrumentation shielding, noise measurements, device characterizations. Magnetic materials with high magnetic permeability are commonly adopted for such cases. Two separate physical mechanism participate at determining the electromagnetic shielding in presence of magnetic materials: the "flux shunting" as a consequence of the high permeability of the shield material and the redirection of flux due to induced eddy currents (Hoburg; 1995). Both these phenomena together to the magnetic field source characteristics, the geometry of the shield and its relevant position with respect to the magnetic field itself contribute to determine the overall SE of a magnetic shield. Due to the highly nonlinear nature of the adopted magnetic materials, the shielding effectiveness vary with the field strength. Saturation effects as well as change of the equivalent magnetic permeability in presence of combined static and time-varying fields can cause inaccurate field analysis and measurements.

The IEEE Std 299, suggests to determine nonlinear effects by measuring the magnetic SE as a function of source strength. This should be done increasing in $10 \mathrm{~dB}$ steps, nominally $0.1 \mathrm{~W}$ to $1 \mathrm{~W}$ and $10 \mathrm{~W}$ the input power at the transmitting antenna. In particular the standard reports that: If the magnetic SE decreases more than about $2 \mathrm{~dB}$, then intermediate level measurements shall be made. The results shall then be plotted to determine the highest level permissible for linear performance (within $\pm 1 \mathrm{~dB}$ ).

Recent hysteresis models have reached a high level of accuracy, as a consequence this makes possible to perform the SE analysis and measurement of a ferromagnetic shield through an accurate characterization of the magnetic behavior of the shielding material combined to a computational analysis (Bologna et al.; 2006; Celozzi \& D'Amore; 1996; Di Fraia et al.; 2009; Sergeant et al.; 2006). 
In particular, in (Di Fraia et al.; 2009) the authors studied an basic case of an iron hollow cylinder placed coaxially around a circular loop. The investigated geometry is shown in Fig. 5

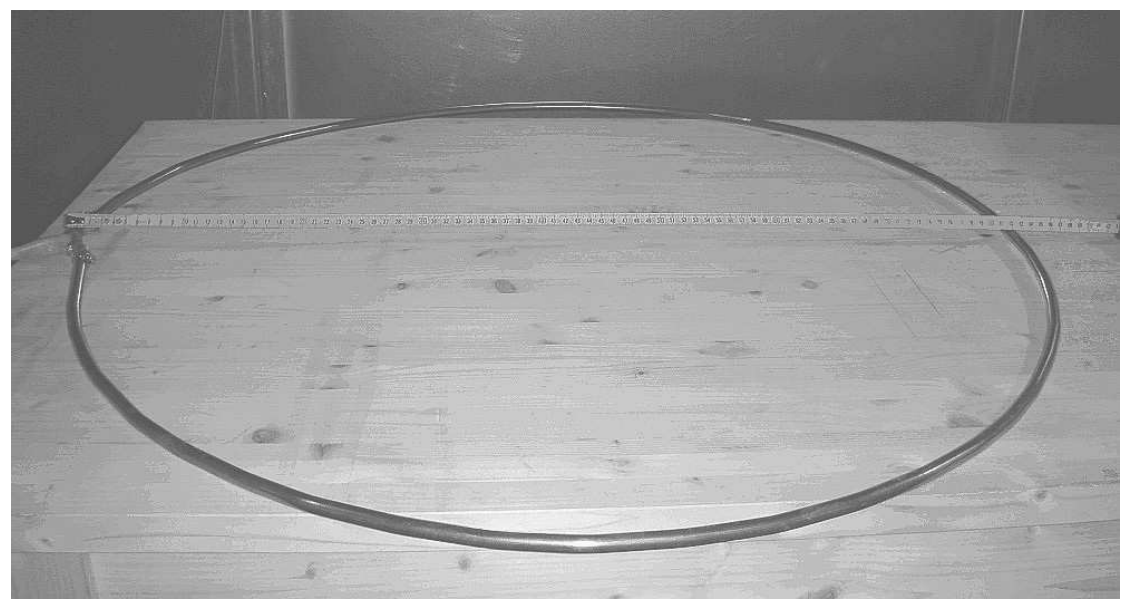

Fig. 5. Photograph of a basic setup for magnetic shield characterization.

The analysis was performed by combining magnetic characterization of the material with an analytical techniques and results were in good agreement with the measurements performed according to the standard procedure (Tellini et al.; 2005).

\section{Magnetic material characterization: data acquisition systems}

The expression "magnetic material characterization" commonly refers to experiments aimed at investigating the dependence of the macroscopic magnetization $M$ vs. the effective magnetic field $H$ in continuos media. In such a way the relationship $M(H)$ has a meaning at the macroscopic level, i.e., $M$ represents the average magnetic moment over a representative spatial region of the material or over the whole test specimen.

Generally speaking an hysteresis loop is interpreted as a property of the material under test. On the other hand, many parameters among which the specimen geometry can influence the measurement and the resulting measured hysteresis cycle. The field $H$ and Mare vector quantities and, strictly speaking, any representation of hysteresis loops should be given in vector terms. However, many experiments and interpretations are based on a scalar representation, where the magnetization component along the field is given as a function of the field intensity. This description is useful and convenient when we can identify an "easy" direction of the fields along the magnetic sample and it is the approach used in this chapter. Of course, this method is not complete being neglected any consideration on the magnetization components perpendicular to the field. The use of vector hysteresis modeling and measurement should be otherwise mandatory for generic magnetic shield geometries. One of the problems we have to face planning experiments of magnetic material characterization is the role of the demagnetizing fields. Such field occurs any time we have a discontinuity of the magnetization vector $M(\nabla \cdot M=0)$ and can influence the measuring methodology and accuracy of the results. Let us consider the basic example shown in Fig. 6 . 
A current $i_{1}(t)$ is driven through a primary coil generating a proportional magnetic field $H_{a}$. The voltage $v_{2}(t)$ induced along a secondary open circuit coil is proportional to the rate of change of the flux $\Phi=B S$, being $S$ the cross-section area of the sample to which is linked the coil and $B$ the average induction component perpendicular to the cross section).
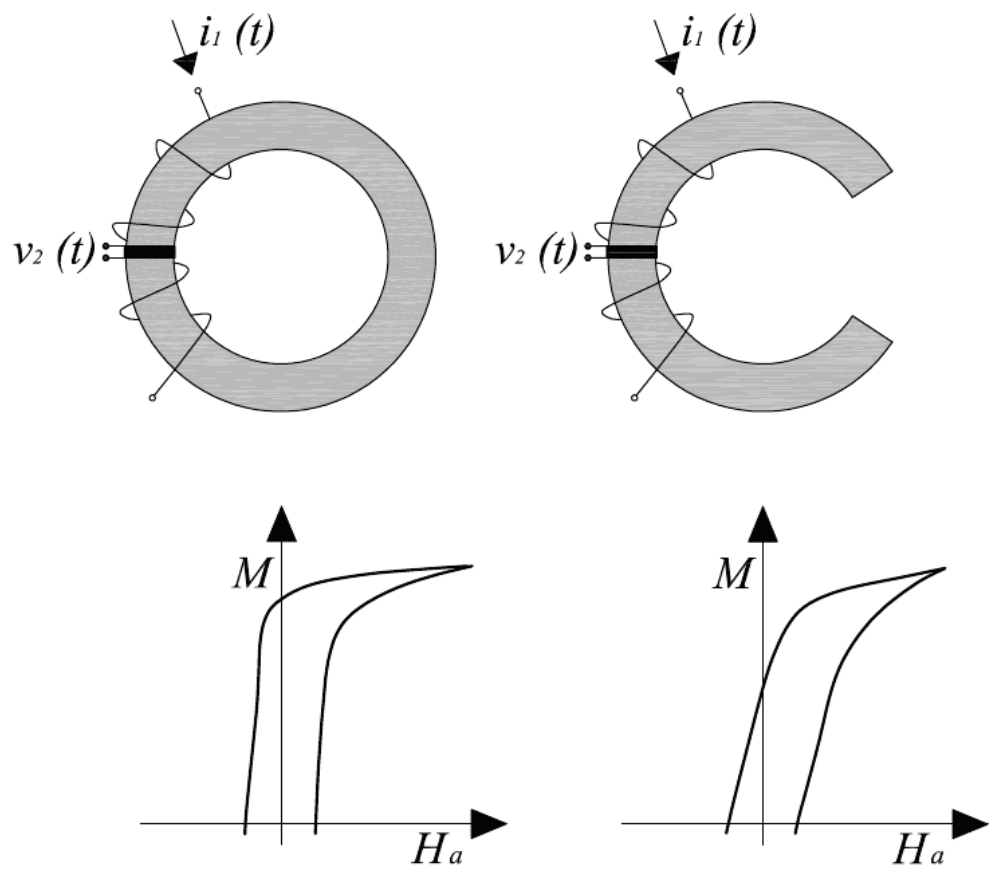

Fig. 6. Schematic representation of open and closed magnetic samples. The loop shape is affected by the specimen geometry.

The applied field $H_{a}$ arising from the primary current $i_{1}(t)$ is the magnetic field that would be present inside the primary coil if magnetic materials were not inserted. In presence of the magnetic material, even driving the same current $i_{1}(t)$ the relationship between $H$ and $i_{1}(t)$ obviously changes. This is easy to show, making two different experiments with the same magnetic material but adopting different specimen geometries, such as those shown in Fig. 6. As discussed in (Bertotti; 1998; Fiorillo; 2004) with the open sample configuration the effective field $H$ acting in the material is not the applied field $H_{a}$ related to the primary current and the demagnetizing field must be taken into account for the characterization of the magnetic material properties.

For such reasons often closed magnetic circuit are preferred to open samples in the measurements of magnetic hysteresis. The sample can be shaped in order to achieve flux closure with the material itself or with a yoke made of a high-permeability and high-section material. An intuitive closed magnetic circuit is represented by the toroidal configuration; some examples are reported in Fig. 7. 

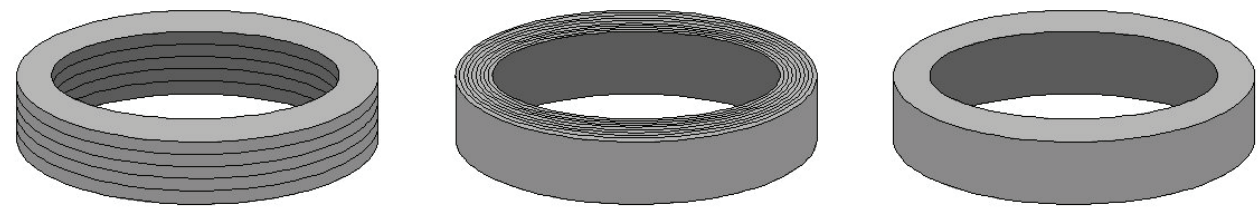

Fig. 7. Schematic representation of different made magnetic cores. Laminated (left one), wound ribbon (center), sintered powder (right one)

Cores can be obtained by stacking rings punched out of a lamination (left one), winding a ribbon-like sample (centre one) or sintering a magnetic powder (right one). The field inside the core can be generated by an uniformly primary winding ound around the core or, if an high intensity field is required, by means of an axial conductor of large cross-sectional area. The stacked specimen can be used in the case of isotropic (or with moderate anisotropy) materials like, for example, non-oriented electrical steels; in this case the magnetic properties measured are averaged over the lamination plane. Strip-wound cores, on the contrary, are used to provide magnetic properties over a definite direction in the plane of the sheet. In every case, using a toroidal core, some aspects must be taken into account:

- the preparation of the specimen and of the primary and secondary winding can be tedious;

- $\quad$ every next specimen requires the preparation of new windings;

- when we want to characterize a lamination along a definite direction a strip-wound core has to be built and this configuration implies the creation of bending stresses that can modify the results;

- the field strength available with the primary winding is limited and we can test only very soft magnets;

- the applied field decreases passing from the inside to the outside boundary of the toroid, being the field strength inversely proportional to the magnetic path length $\mathrm{D}$ (where $\mathrm{D}$ is the diameter of the considered circumferential field line). This leads to geometrical constraints among the internal and external diameter.

Such drawbacks typical of toroidal specimen brought to the development of a different closed magnetic circuit obtained by making a square assembly of strips cut along the desired testing direction, superimposed at the corners. The specimen is known as the Epstein test frame and such a magnetic configuration is a standard for measurements in steel sheets from DC to $10 \mathrm{kHz}$. A multiple number of four strips of width $30 \mathrm{~mm}$ and length variable from 280 to $305 \mathrm{~mm}$ are superposed at the corner to forma square. A weight of $1 \mathrm{~N}$ is placed on each corner in order to ensure a good and reproducible flux closure. Each side of the square is provided with a secondary and a primary winding wound on a rigid insulation support with rectangular section. The solenoids have all the same number of turns with a total of 700 primary and secondary turns in the frame used for DC and power frequency measurements and a total of 200 turns in the frame recommended for medium frequency range $(0.4-10 \mathrm{kHz})$ (IEC 60404-2; 2008; IEC 60404-10; 1988). The mean magnetic path is fixed by the standard IEC 60404-2 in 0.94 m. In Figs. 8 and 9 a schematic drawing and a photograph of an Epstein frame are reported. 


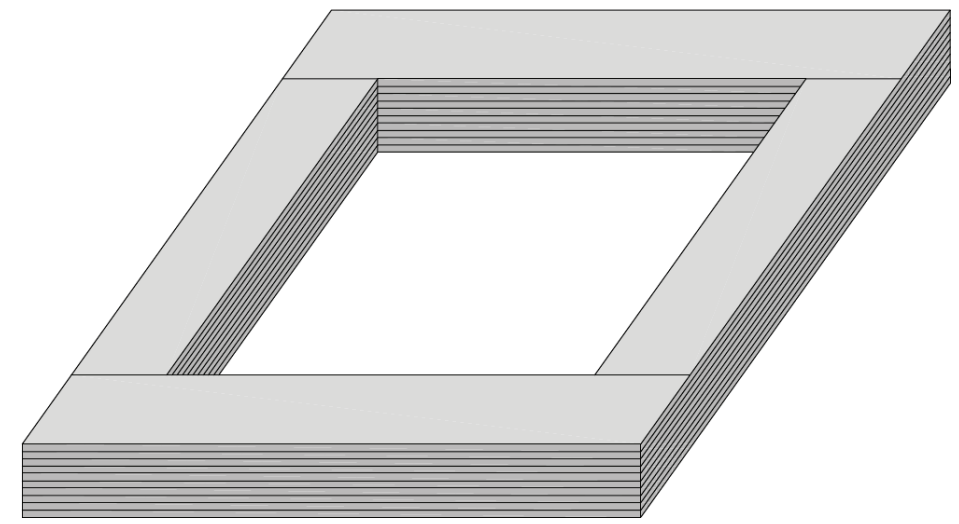

Fig. 8. Schematic drawing of the Epstein frame.

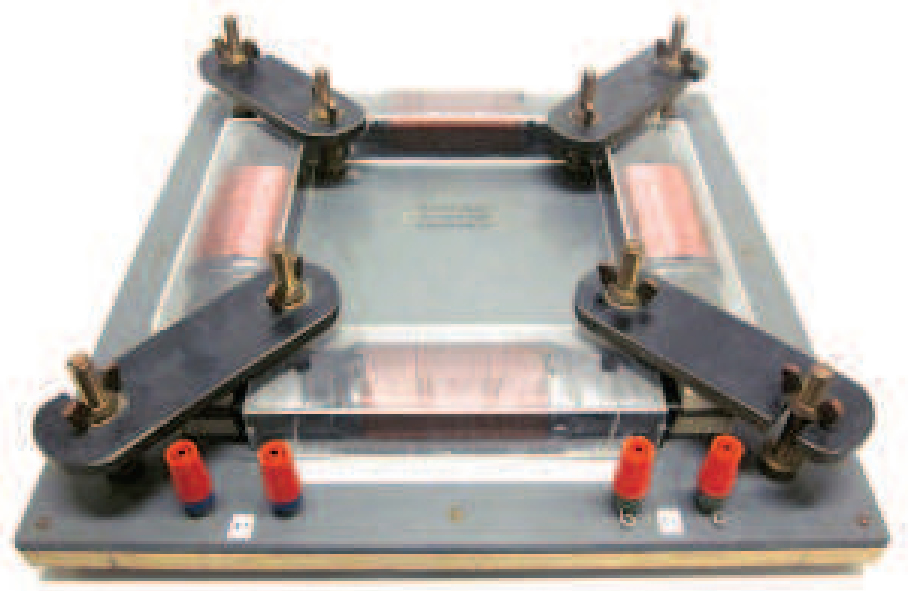

Fig. 9. Photograph of the Epstein frame.

A measurement technique commonly adopted for characterization of soft magnetic materials is the volt-amperometric method. This approach reconstructs the magnetic $H$ and induction $B$ fields via the measurement of the primary current $i_{1}$ and the secondary open circuit voltage $v_{2}$ through the Ampère and Lenz's laws:

$$
H=\frac{N_{1} i_{1}}{L_{m}} \quad v_{2}=N_{2} S \frac{d B}{d t}
$$

where $N_{1}$ and $N_{2}$ represent the number of turns in the primary and secondary winding (700 each one in the case of the Epstein frame used for DC and power frequency measurements); $L m$ is the mean magnetic path length ( $0.94 \mathrm{~m}$ in the case of the Epstein frame), and $S$ is the cross section area of the magnetic circuit. The volt-amperometric method is generally applied for measuring of major loop or symmetric minor loops. Recently this techniques has been extended to the characterization of asymmetric minor loops and of magnetic materials under nonperiodic conditions (Tellini et al.; 2008, 2009). 
In Fig. 10 a basic measurement scheme for characterization of soft magnetic materials is reported.

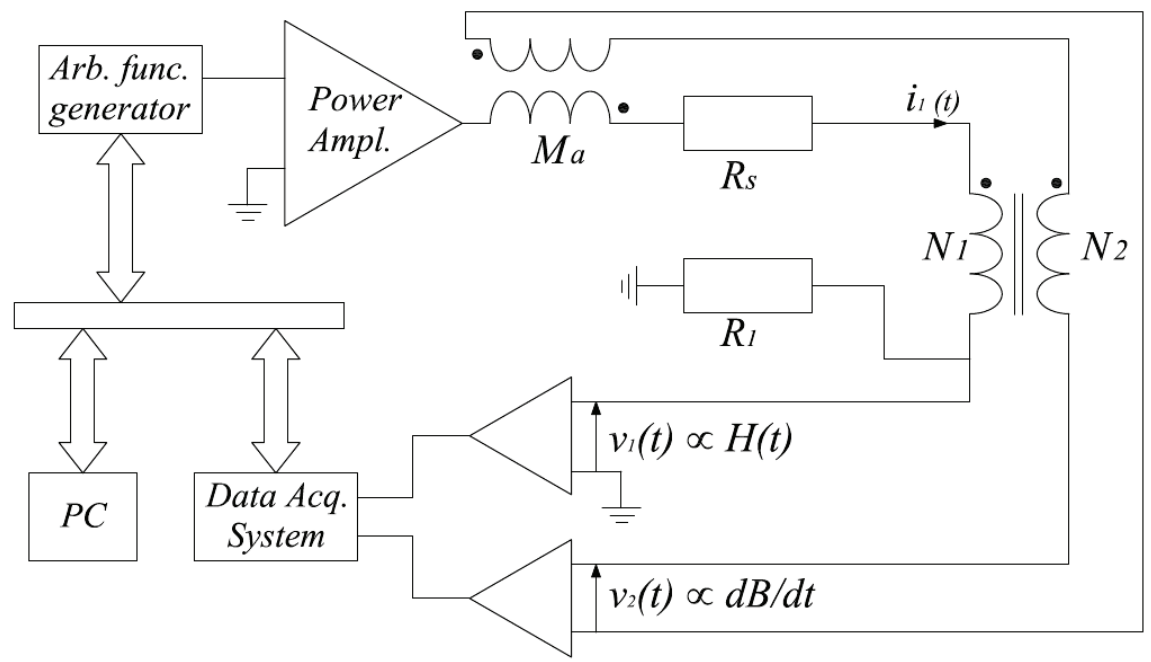

Fig. 10. Measurement scheme for characterization of soft magnetic materials.

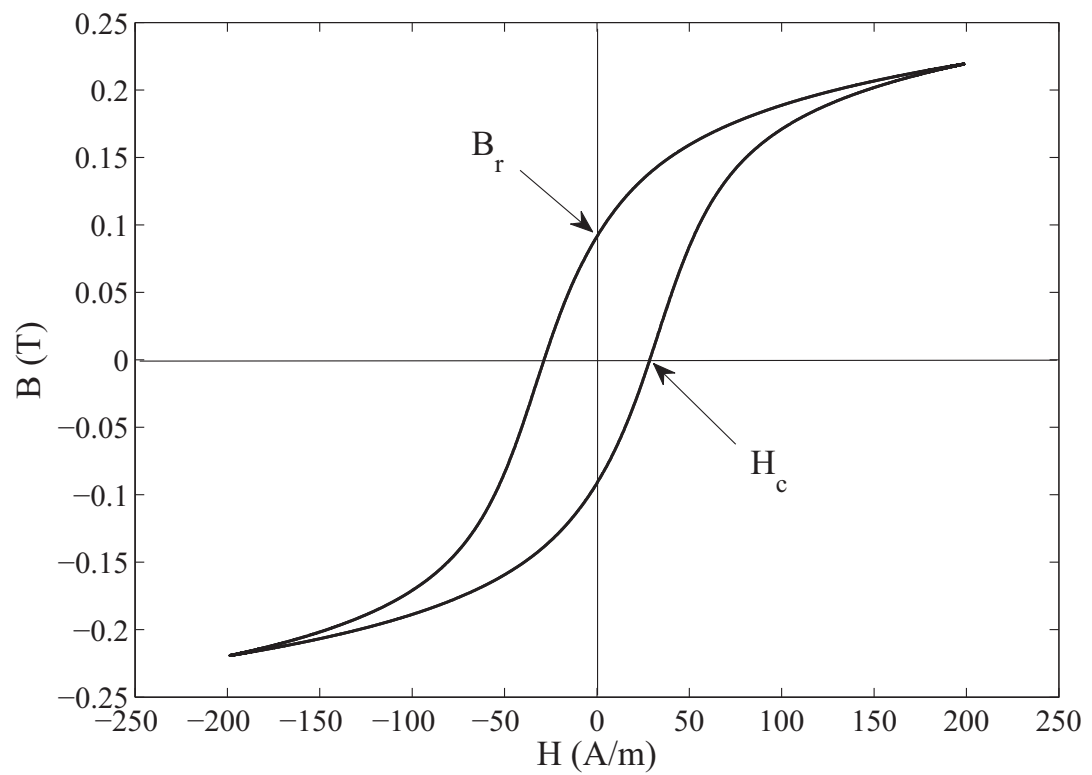

Fig. 11. Major loop obtained for a commercial soft ferrite. Coercive field $H c$ and induction remanence $\mathrm{Br}$ are indicated by arrows. 
An arbitrary function generator is connected to the primary winding via a power amplifier. The primary current $i_{1}$ is measured via the voltage drop across a calibrated resistance $R_{H}$. The secondary open circuit voltage $v_{2}$ is measured by means of a high-impedance differential amplifier and a data acquisition system.

The data acquisition system must perform synchronous acquisitions between the two channels; a couple of identical DC-coupled variable-gain low-noise amplifiers is generally interposed between the $H(t)$ and $d B / d t$ signal sources and the acquisition device (Fiorillo; 2004).

The mutual inductance $M_{a}$ in the scheme of Fig. 10 is used to automatically compensate the air flux linked with the secondary winding. The presented scheme can be used to impose a prescribed time dependence (often sinusoidal) of the magnetization, i.e. the secondary voltage $v_{2}(t)$ for example by means of a digitally controlled recursive technique.

In Fig. 11, the hysteresis loop obtained measuring data on a commercial ferrite toroid is reported. Although a complete model of magnetic hysteresis is very complex, the coercive field $H_{c}$ and the induction remanence $B_{r}$ are two key paramaters that together to the saturation $H_{\text {sat }}, B_{\text {sat }}$ values define in a first approximation the material magnetic behavior. The remanence $B_{r}$ represents the induction value obtained after applying a large field to the specimen and then removing it, while the coercive field is the field needed to bring the induction field from $B_{r}$ to zero. On the basis of $H_{c}$ and $B_{r}$ values, magnetic materials are commonly classified into soft and hardmagnetic materials. In Fig. 12 a typical major loop with a complete series of minor symmetric cycles is shown. Such data are a basic set for the identification of scalar hysteresis models such as the Preisach scalar model Cardelli et al. (2000). The magnetic sample under test was a commercial ferrite toroidal specimen.

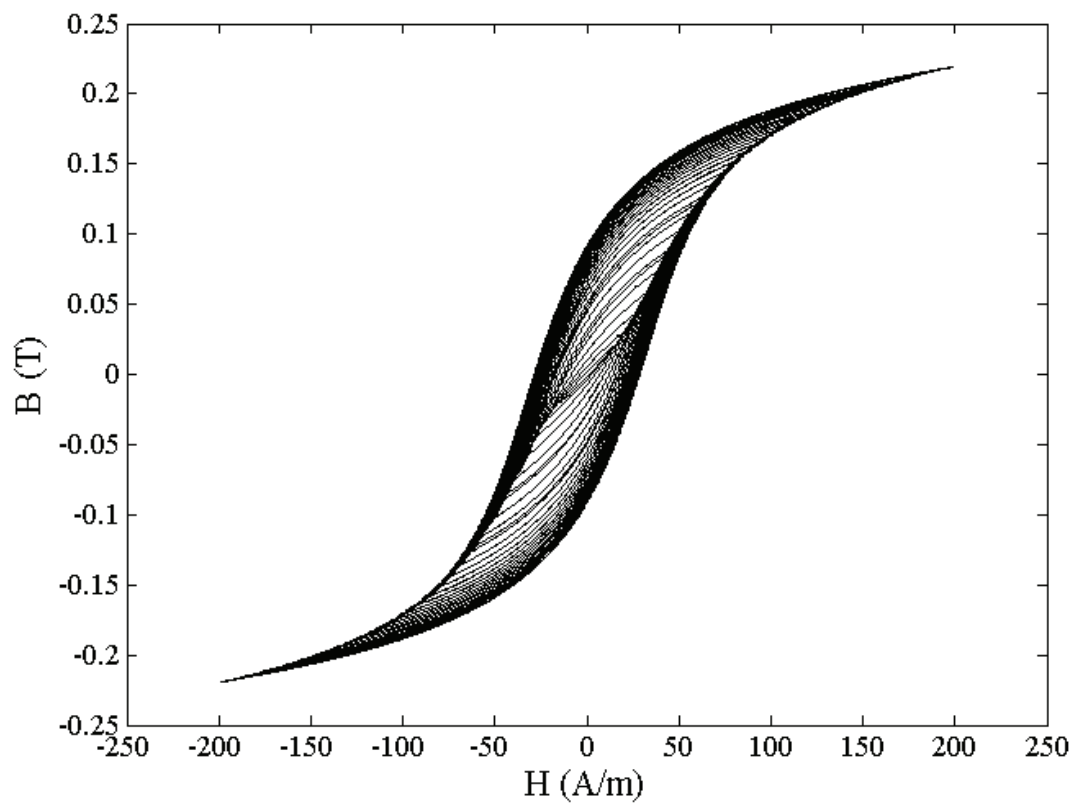

Fig. 12. Major loop and minor symmetric cycles obtained for a commercial soft ferrite. 


\section{Conclusions}

The chapter presented basic aspects of the shielding theory and shielding effectiveness measurement. In a first part, some remarks were spent on the classical eddy current analysis and the impedance concept (Schelkunoff's theory) for approaching shielding problems. In a second part, the discussion was oriented towards common and alternative measurement procedures. In particular, time-frequency or pulsed signal based measurement techniques were described as possible effective tools for application to dispersive or non-linear shielding materials. The third and last part focused on the magnetic shields and on the characterization procedures of the magnetic materials. The discussion points out the importance of an accurate knowledge of the material magnetic behavior in order to improve the shielding design and to make more efficient the measurements of the shielding parameters.

\section{References}

Angrisani L.; Daponte P. \& D'Apuzzo M. (2000) A measurement method based on time frequency representations for testing GSM equipment, IEEE Trans. on Instr. and Meas., vol.49, No.5, October 2000, pp.1050-1055.

Angrisani L.; \& D'Arco M. (2002) A measurement method based on an modified version of the chirplet transform for instantaneous frequency estimation, IEEE Trans. on Instr. and Meas., vol.51, No.4, August 2002, pp.704-711.

Bertotti, G. (1998). Hysteresis in Magnetism: For Physicists, Materials Scientists, and Engineers, Academic Press.

Bologna, M.; Giannetti, R.; Marracci, M. \& Tellini, B. (2006). Measuring the Magnetic Field Attenuation of Nonlinear Shields," IMTC Conference, (2006), 2200-2204.

Braun, S.; Donauer, T. \& Russer, P. (2008). A Real-Time Time-Domain EMI Measurement System for Full-ComplianceMeasurements According to CISPR 16-1-1. IEEE Trans. Electromag. Compat., Vol. 50, No. 2, (May 2008), 259-267.

Cardelli, E.; Della Torre, E.; Tellini, B. (2000). Direct and Inverse Preisach Modelling of Soft Materials. IEEE Trans. Magn., Vol. 36, No. 4, (Jul. 2000), 1267-1271.

Celozzi, S. \& D'Amore, M. (1996). Magnetic Field Attenuation of Nonlinear Shields. IEEE Trans. Electromag. Compat., Vol. 38, No. 3, (Aug 1996), 318-326.

Di Fraia, S.;Marracci,M.; Tellini, B. \& Zappacosta, C. (2009). Shielding EffectivenessMeasurements for Ferromagnetic Shields. IEEE Trans. Instrum.Meas., Vol. 58, No. 1, (Jan 2009), 115-121.

Fiorillo, F. (2004). Measurement and Characterization of Magnetic Materials, Elsevier-Academic Press.

Hlawatsch, F. \& Boudreaux-Bartels, G.F. (1992). Linear and Quadratic Time-Frequency Signal Representation, IEEE Signal Processing Magazine, April 1992.

Hoburg J. F. (1988). Principles of Quasistatic Magnetic Shielding with Cylindrical and Spherical Shields. IEEE Trans. Electromag. Compat., Vol. 37, No. 4, (Nov 1995), 574579.

IEC 60404-2 (2008). Magnetic materials - Part 2: Methods of measurement of the magnetic properties of electrical steel strip and sheet by means of an Epstein frame.

IEC 60404-10 (1988).Magneticmaterials - Part 10: Methods ofmeasurement ofmagnetic properties of magnetic sheet and strip at medium frequencies. 
IEEE Std 299 (2006). IEEE Standard Method for Measuring the Effectiveness of Electromagnetic Shielding Enclosures.

IEEE Std 393-1991 (1992). IEEE Standard for Test Procedures for Magnetic Cores.

Krug, F. \& Russer, P. (2005). Quasi-Peak Detector Model for a Time-Domain Measurement System. IEEE Trans. Electromag. Compat., Vol. 47, No. 2, (May 2005), 320-326.

Moser J. R. (1988). Low-Frequency Low-Impedance Electromagnetic Shielding. IEEE Trans. Electromag. Compat., Vol. 30, No. 3, (Aug 1988), 202-210.

NIST Technical Note 1297 (1994). Guidelines for Evaluating and Expressing the Uncertainty of NIST Measurement Results. Barry N. Taylor and Chris E. Kuyatt.

Paul, C. R. (1992). Introduction to Electromagnetic Compatibility,Wiley, NewYork.

Schelkunoff, S. A. (1943). Electromagnetic Waves, Princeton, NJ, Van Nostrand.

Schulz, R. B.; Plantz, V. C. \& Brush D. R. (1988). Shielding Theory and Practice. IEEE Trans. Electromag. Compat., Vol. 30, No. 3, (Aug 1988), 187-201.

Sergeant P.; Zucca, M.; Dupré, L. \& Roccato, P. E. (2006). Magnetic shielding of a cylindrical shield in nonlinear hystereticmaterial. IEEE Trans.Magn., Vol. 42, No. 10, (Oct. 2001), 3189-3191.

Tellini, B.; Bologna, M. \& Pelliccia, D. (2005). A new analytic approach for dealing with hysteretic materials. IEEE Trans.Magn., Vol. 41, No. 1, (Jan. 2005), 2-7.

Tellini, B.; Giannetti, R. \& Lizón-Martínez, S. (2008). Sensorless Measurement Technique for Characterization of Magnetic Materials under Nonperiodic Conditions. IEEE Trans. Instrum. Meas., Vol. 57, No. 7, (July 2008), 1465-1469.

Tellini, B.; Giannetti, R.; Lizón-Martínez, S. \& Marracci, M. (2009). Characterization of the Accommodation Effect in Soft Hysteretic Materials via Sensorless Measurement Technique. IEEE Trans. Instrum.Meas., Vol. 58, No. 10, (Aug. 2009), 2807-2814.

Tegopoulos, J. A. \& Kriezis E. E. (1985). Eddy Currents in Linear Conducting Media, Elsevier, Amsterdam, Oxford, New York, Tokyo. 


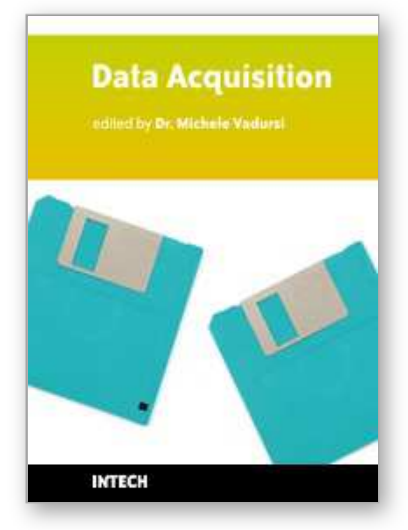

\author{
Data Acquisition \\ Edited by Michele Vadursi
}

ISBN 978-953-307-193-0

Hard cover, 344 pages

Publisher Sciyo

Published online 28, September, 2010

Published in print edition September, 2010

The book is intended to be a collection of contributions providing a birdâ€ ${ }^{\mathrm{TM}} \mathrm{s}$ eye view of some relevant multidisciplinary applications of data acquisition. While assuming that the reader is familiar with the basics of sampling theory and analog-to-digital conversion, the attention is focused on applied research and industrial applications of data acquisition. Even in the few cases when theoretical issues are investigated, the goal is making the theory comprehensible to a wide, application- oriented, audience.

\title{
How to reference
}

In order to correctly reference this scholarly work, feel free to copy and paste the following:

Leopoldo Angrisani, Mirko Marracci, Nicola Pasquino and Bernardo Tellini (2010). Data Acquisition Systems for Magnetic Shield Characterization, Data Acquisition, Michele Vadursi (Ed.), ISBN: 978-953-307-193-0, InTech, Available from: http://www.intechopen.com/books/data-acquisition/data-acquisition-systems-formagnetic-shield-characterization

\section{INTECH}

open science | open minds

\author{
InTech Europe \\ University Campus STeP Ri \\ Slavka Krautzeka 83/A \\ 51000 Rijeka, Croatia \\ Phone: +385 (51) 770447 \\ Fax: +385 (51) 686166 \\ www.intechopen.com
}

\author{
InTech China \\ Unit 405, Office Block, Hotel Equatorial Shanghai \\ No.65, Yan An Road (West), Shanghai, 200040, China \\ 中国上海市延安西路65号上海国际贵都大饭店办公楼405单元 \\ Phone: +86-21-62489820 \\ Fax: +86-21-62489821
}


(C) 2010 The Author(s). Licensee IntechOpen. This chapter is distributed under the terms of the Creative Commons Attribution-NonCommercialShareAlike-3.0 License, which permits use, distribution and reproduction for non-commercial purposes, provided the original is properly cited and derivative works building on this content are distributed under the same license. 